\title{
Research on Operating Characteristics of Two-Terminal Flexible Direct Transmission with Improved Deadbeat Control
}

\author{
Mei Jianchun ${ }^{1}$, Qian Junxia ${ }^{1}$, Zhao Shengjie ${ }^{1}$, Ma Hongjuan ${ }^{1}$, Song Pengcheng ${ }^{1}$, Shen Zhoupeng ${ }^{2 *}$ \\ ${ }^{1}$ Jiangsu Keneng Electric Power Engineering Cosulting Co. LTD \\ ${ }^{2}$ Nanjing Normal University. School of Electrical and Automation Engineering, Nanjing Normal University, Nanjing 210042, China
}

\begin{abstract}
Traditional DC transmission technology has obvious defects in power adjustment speed, loss reduction, protection and control, etc., and it has been unable to meet the diverse needs of electricity consumption. The flexible DC transmission system not only solves the above shortcomings, but also has the advantages of independently controllable power and power supply to passive network. It is widely applied in distributed power generation by new energy, micro grid and other fields. This paper takes the two-terminal flexible DC transmission system as the research object, establishes the mathematical mode of two-terminal flexible DC transmission system, and studies the basic operation mode of the system and the voltage source converter (VSC) control strategy on this basis. Considering the problems of control delay and poor responsiveness of current closed-loop control when the traditional deadbeat control is applied to VSC converters, an improved deadbeat control strategy is proposed to solve the above-mentioned defects by predicting the current sampling at k+2 moment. Finally, the co-simulation is used to verify the correctness and reliability of the above-mentioned method.
\end{abstract}

\section{Introduction}

With the increasing pollution of the environment, distributed energy sources such as wind energy and solar energy have become research hotspots due to the advantages of environmental protection, renewable, and flexible operation. However, their defects are also very prominent, such as intermittent power fluctuations, far away from power centers, small scale and scattered distribution. In addition, the population of our country is growing, the electricity consumption in cities is constantly rising, and the transformation and expansion of the urban power supply network has become increasingly important $[1-2]$. With the application of fully-controlled new semiconductor devices such as IGBT and GTO in the 1990s replacing traditional semi-controlled switching devices in HVDC transmission projects on a large scale, major breakthroughs have been made in the structure and control strategy of the HVDC transmission system. Instead, the flexible direct current transmission technology (VSC-HVDC) is widely applied in practical projects, which is mainly composed of voltage source converters, PWM modulation technology, etc., In distributed energy grid connection, passive network power supply, urban areas power supply transformation and other occasions VSC-HVDC has irreplaceable advantages, which can better solve the above-mentioned problems [3-5].

At present, domestic and foreign scholars have done a lot of research on flexible direct current transmission technology (VSC-HVDC), mainly focusing on system controller design and VSC control strategy. Literature [6] established a steady-state model based on the VSC flexible DC transmission system, and designed a nonlinear controller composed of inverse model and PI controller. Literature [7] designed active power and constant voltage controllers and constant $\mathrm{DC}$ voltage and constant AC voltage controllers for the active and passive networks connected to the system. In literature [8], in order to shorten the state switching process between multiple converters, an improved droop control strategy is adopted to shorten the switching time and ensure the stability of the system DC voltage and the balanced power distribution. Literature [9-10] designed constant DC voltage and constant $\mathrm{AC}$ voltage controllers for passive power supply networks, and simulated various control conditions through simulation. Literature [11] adopted deadbeat control to suppress the system resonance, but it combines the state observer to design the system, whose control algorithm is too complicated, and it is difficult to implement in the actual system.

This paper takes the two-terminal flexible direct current transmission system as the research object, establishes the two-terminal flexible direct current mathematical model, and studies the basic operation mode of the system and the voltage source converter (VSC) control strategy on this basis. Considering the problems of control delay and poor responsiveness of current closedloop control when the traditional deadbeat control is applied to VSC converters, an improved deadbeat control strategy is proposed to solve the above-mentioned defects

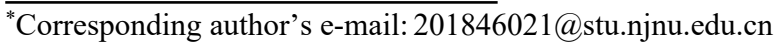


by predicting the current sampling at $\mathrm{k}+2$. Finally, the cosimulation is used to verify the correctness and reliability of the above-mentioned method.

\section{Mathematical mode of two-terminal flexible DC transmission system}

As shown in Figure 1, the two-terminal flexible DC is composed of a converter station 1, a DC side capacitor and a converter station 2 . The DC side capacitor provides a stable DC voltage for the system to ensure active and reactive power flow, and the reactor $\left(X_{L i}\right)$ is used to filter the harmonics of the input and output current at the rectifier end and the inverter end. When analyzing the system in this paper, in order to facilitate the derivation of the mathematical model, the parallel loss of the converter is ignored, and the series loss of the converter and other losses are represented by the equivalent resistance $R_{i}$ [12-15].

\subsection{Two-terminal flexible DC mathematical model in three-phase coordinate system}

As shown in Figure 1, the dynamic differential equation of the flexible back-to-back HVDC transmission system in the three-phase static coordinate system can be obtained by drawing the KCL equation:

$$
\left\{\begin{array}{l}
L \frac{d i_{j 1}}{d t}+R i_{j 1}=E_{s j}-E_{j} \\
L \frac{d i_{j 2}}{d t}+R i_{j 2}=V_{j}-V_{s j}
\end{array} \quad(j=a, b, c)\right.
$$

In the formula, $i_{j 1}$ and $i_{j 2}$ are the input and output current of the AC system $E_{s j}$ and $V_{s j}$ are input and output voltages of the AC system. $E_{j}$ and $V_{j}$ are the input and output voltages on the AC side of converter 1 and converter 2.Because the structural parameters of converter 1 and converter 2 are symmetric, only the switching function of three-phase bridge arm of converter 1 is defined:

$$
\left\{\begin{array}{l}
m_{a 1}=s_{a}-\frac{1}{3} \sum_{j=a, b, c} s_{j} \\
m_{b 1}=s_{b}-\frac{1}{3} \sum_{j=a, b, c} s_{j} \\
m_{c 1}=s_{c}-\frac{1}{3} \sum_{j=a, b, c} s_{j}
\end{array}\right.
$$

Substituting Equation (2) into Equation (1), we get:

$$
\left\{\begin{array}{l}
L \frac{d i_{j 1}}{d t}+R i_{j 1}=E_{s j}-u_{d c} m_{j 1} \\
L \frac{d i_{j 2}}{d t}+R i_{j 2}=u_{d c} m_{j 2}-V_{s j}
\end{array} \quad(j=a, b, c)\right.
$$

Figure 1 DC-side voltage equation can be obtained as follows:

$$
\begin{aligned}
& C \frac{d u_{d c}}{d t}=i_{01}-i_{02}=\left(m_{a 1} i_{a 1}+m_{b 1} i_{b 1}+m_{c 1} i_{c 1}\right)- \\
& \left(m_{a 2} i_{a 2}+m_{b 2} i_{b 2}+m_{c 2} i_{c 2}\right)
\end{aligned}
$$

$u_{d c}$ is the DC side voltage; $i_{01}$ and $i_{02}$ are DC input or output current. Assuming that the switching loss of the converter is ignored, we can get:

$$
\left\{\begin{array}{l}
P_{c 1}=E_{a} i_{a 1}+E_{b} i_{b 1}+E_{c} i_{c 1}= \\
u_{d c}\left(m_{a 1} i_{a 1}+m_{b 1} i_{b 1}+m_{c 1} i_{c 1}\right)=u_{d c} i_{01} \\
P_{c 2}=V_{a} i_{a 1}+V_{b} i_{b 1}+V_{c} i_{c 1}= \\
u_{d c}\left(m_{a 2} i_{a 2}+m_{b 2} i_{b 2}+m_{c 2} i_{c 2}\right)=u_{d c} i_{02}
\end{array}\right.
$$

In the formula, $P_{c 1}$ and $P_{c 2}$ are AC output or input active power of converter 1 and converter 2 respectively. When $u_{d c}$ in dc side is constant, the system is in the stable operation state at this time, $i_{01}=i_{02}, P_{c 1}=P_{c 2}$, namely input power equals to output power. It can be concluded that to achieve the power balance on both sides of the flexible back-to-back, it is necessary to ensure the stability of the DC side voltage.

Equation (3) constitutes the mathematical model of the two-terminal flexible DC system; Equation (4) constitutes the mathematical model of the DC side voltage; Equation (3) and (4) constitute the mathematical model of the twoterminal flexible DC system in the three-phase static coordinate system.

Since the parameters in the three-phase static coordinate system are all variable AC quantities, which is not conducive to the system control and design, the twoterminal flexible DC mathematical model must be transformed into the synchronous rotating DQ coordinate system, and the sine variable in the three-phase static coordinate system must be transformed into the direct flow in the synchronous coordinate system.

\subsection{Two-terminal flexible DC mathematical model in $D Q$ coordinate system}

According to Equations (3) and (4), the mathematical model of the two-terminal flexible DC in the DQ synchronous rotation coordinate system is established:

$$
\begin{aligned}
& \frac{d}{d t}\left[\begin{array}{l}
i_{d 1} \\
i_{q 1}
\end{array}\right]=\left[\begin{array}{cc}
-\frac{R}{L} & \omega_{1} \\
-\omega_{1} & -\frac{R}{L}
\end{array}\right]\left[\begin{array}{l}
i_{d 1} \\
i_{q 1}
\end{array}\right]-\left[\begin{array}{l}
\frac{u_{d c} m_{d 1}-E_{s d}}{L} \\
\frac{u_{d c} m_{q 1}}{L}
\end{array}\right] \\
& \frac{d}{d t}\left[\begin{array}{l}
i_{d 2} \\
i_{q 2}
\end{array}\right]=\left[\begin{array}{ll}
-\frac{R}{L} & \omega_{2} \\
-\omega_{2} & -\frac{R}{L}
\end{array}\right]\left[\begin{array}{l}
i_{d 2} \\
i_{q 2}
\end{array}\right]-\left[\begin{array}{l}
\frac{V_{s d}-u_{d c} m_{d 2}}{L} \\
-\frac{u_{d c} m_{q 2}}{L}
\end{array}\right]
\end{aligned}
$$

The equation of the DC side voltage in the rotation coordinate of $\mathrm{dq}$ is as follows:

$$
\frac{d u_{d c}}{d t}=\frac{m_{d 1} i_{d 1}+m_{q 1} i_{q 1}}{C}-\frac{m_{d 2} i_{d 2}+m_{q 2} i_{q 2}}{C}
$$


Above all: $\omega_{1}, \omega_{2}$ is the angular frequency of the phase voltage ac system; $i_{d 1}, i_{d 2}, i_{q 1}$ and $i_{q 2}$ are the components of the three-phase AC current of the AC system on the DQ axis; $E_{s d}$ and $V_{s d}$ are the components of the three-phase voltage of the AC system at both ends on the D-axis. $m_{d 1}, m_{d 2}, m_{q 1}$ and $m_{q 2}$ are the components of the converter switching function on the DQ axis. Equations (6), (7) and (8) together constitute the mathematical model of two-terminal flexible DC system in the DQ rotation coordinate system.

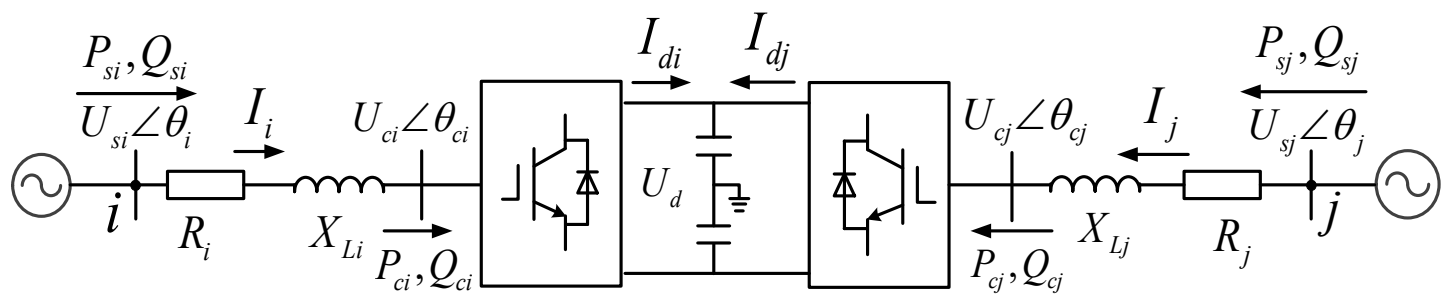

Figure 1. Flexible DC transmission main circuit structure.

\section{0peration analysis based on improved deadbeat system}

\subsection{Basic control mode of the system}

According to the research of existing scholars and practical engineering applications, two-terminal flexible DC control is generally divided into two situations: 1 . Control mode when both ends are connected to the grid; 2 . Control mode when supplying power to passive network. Control mode when both ends are connected to the grid :(1) Constant DC voltage control mode, used to stabilize the DC capacitance voltage and balance the power flow at both ends;(2) Constant DC current (power) control mode is used to control the flow of active power and balance the flow of power flow at both ends. Control mode of power supply to passive network :(1) AC voltage control mode is fixed to stabilize the AC output voltage of the inverter end and the passive network side; Generally speaking, for flexible flexible systems with two ends, one end must adopt constant DC voltage control mode to support the system voltage, and the other end must adopt constant DC current (power) or constant $\mathrm{AC}$ voltage depending on whether the network connected is active or passive.

\subsection{Traditional deadbeat control strategy}

Deadbeat control is a control system that combines the system state equation, the system output feedback equation, and the required next-moment reference output equation to calculate the required switching pulses. Compared with other VSC control strategies, it has the advantages of fixed switching frequency, fast dynamic response and high bandwidth, etc., and has been widely used in practice [16].In a digital system, the reference output of the system at the next moment needs to be expressed as the relationship between the system state variable and the output feedback variable in order to achieve the control effect of the deadbeat. According to the type (6), (7), because of $\alpha \beta$ axis equation is symmetrical, so if we take $\alpha$ axis for example, deadbeat control block diagram in $\alpha$ axis can be gotten. As shown in Figure 2, $G_{\mathrm{pwm}}(z)$ is PWM modulation transfer function, $G_{\mathrm{p}}(z)$ is the transfer function of the object.

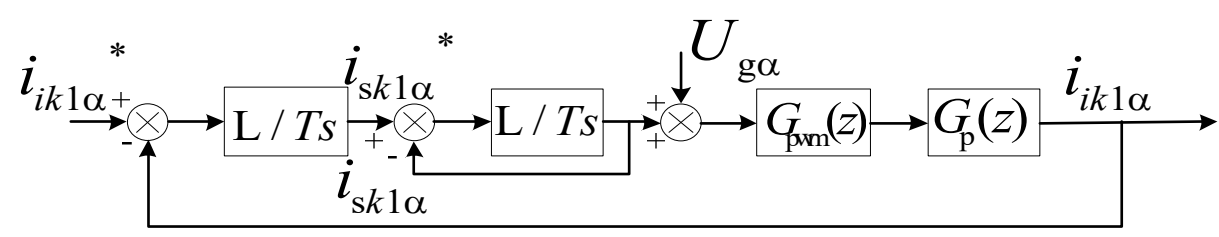

Figure 2. Block diagram of current loop with deadbead control.

As can be seen from Figure 2, the traditional deadbeat current control has actually become one-beat control, and it is impossible to achieve deadbeat tracking. Moreover, due to the limitation of system hardware and controller capacity, the system sampling rate should not be too high. At the same time, considering the application environment of the system, higher control precision and response rate are needed. Therefore, the traditional deadbeat control can no longer meet the requirements of practical engineering application, so it needs to be improved to improve the control effect [17].

\subsection{Improve the deadbeat control strategy}

Due to the limitations of sampling rate and calculation capacity of the actual controller, the traditional deadbeat cannot realize the completion of sampling, calculation and duty cycle output at the same time, and there is always the situation that the sampling point leads the duty cycle output point. Figure 3 is a control delay sketch map, the picture in the first moment, $k$ system still enforce $k-1$ moment PWM duty cycle $\mathrm{D}_{(k-1)}$, while on duty ratio $\mathrm{D}_{(k)}$ 
of a time delay $t_{\mathrm{d}}$ to output. No beat control precision in order to further improve, fixed cyclical deviations due to the above, the improved algorithm by predicting $k+2$ time of current, the PWM duty cycle $\mathrm{D}_{(k+1)}$ can be in $k+1$ moment in advance execution to realize the current astatic tracking.

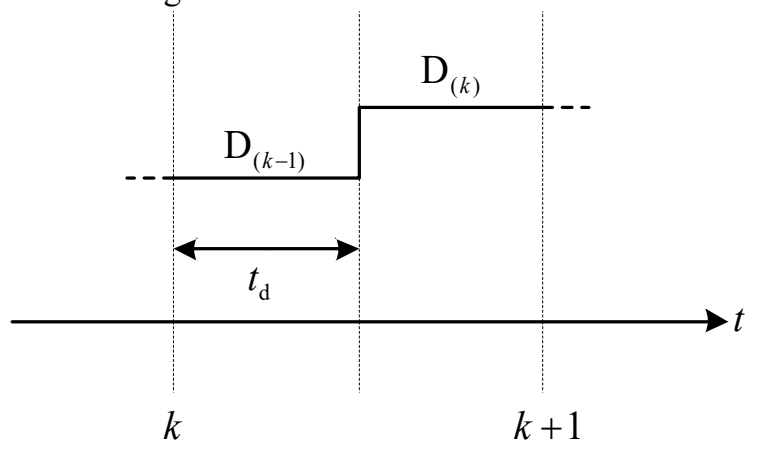

Figure 3. Sketch map of control delay.

By Equation (6), (7) calculate the current value of the next moment, ignore the capacitance of the system, the default current $i_{i k 1}=i_{\mathrm{s} k 1}=i$, then the sampling value in $\alpha$ axis at $k+2$ moment is:

$$
\begin{aligned}
& i_{\alpha}(k+2)=\left(u_{\alpha}(k+1)-U_{\mathrm{g} \alpha}(k+1)\right) . \\
& \frac{T s}{\mathrm{~L}}+i_{\alpha}(k+1)
\end{aligned}
$$

According to the Equation (6), (7) and (9), the current deviation in $\alpha$ axis at $k$ moment and $k+1$ moment is:

$$
\begin{aligned}
& \Delta i_{\alpha}(k)=i_{\alpha}(k+1)-i_{\alpha}(k)=\frac{T s}{\mathrm{~L}} . \\
& \left(u_{\alpha}(k)-U_{\mathrm{g} \alpha}(k)\right) \\
& \Delta i_{\alpha}(k+1)=i_{\alpha}(k+2)-i_{\alpha}(k+1)= \\
& \frac{T s}{\mathrm{~L}}\left(u_{\alpha}(k+1)-U_{\mathrm{g} \alpha}(k+1)\right)
\end{aligned}
$$

Make the current error in $\alpha$ axis at $k+1$ moment is equivalent to two adjacent moment current error of average, the expression is:

$$
\begin{aligned}
& \Delta i_{\alpha}(k+1)=\frac{1}{2}\left(\Delta i_{\alpha}(k)+\Delta i_{\alpha}(k+1)\right) \\
& =\frac{1}{2}\left(i_{\alpha}(k+2)-i_{\alpha}(k)\right)
\end{aligned}
$$

By substituting Equation (12) into Equation (11), the output voltage of the $\mathrm{AC}$ side of the system can be obtained:

$$
\begin{aligned}
& u_{\alpha}(k+1)=U_{\mathrm{g} \alpha}(k+1)+ \\
& \frac{\mathrm{L}}{2 T S}\left(i_{\alpha}(k+2)-i_{\alpha}(k)\right)
\end{aligned}
$$

When considering fundamental frequency to the power grid, if the sampling period is smaller, we can think that the sampling deviation of grid side voltage in $\alpha$ axis in three consecutive sampling time are equal, expression is:

$$
\begin{aligned}
& U_{\mathrm{g} \alpha}(k+1)-U_{\mathrm{g} \alpha}(k)= \\
& U_{\mathrm{g} \alpha}(k)-U_{\mathrm{g} \alpha}(k-1)
\end{aligned}
$$

By Equation (14), the average value of network voltage at $k+1$ moment can be inferenced by linear extrapolation of several sample values, expression is:

$$
\begin{aligned}
& \hat{U}_{\mathrm{g} \alpha}(k+1)=U_{\mathrm{g} \alpha}(k)+\left(U_{\mathrm{g} \alpha}(k+1)\right. \\
& \left.-U_{\mathrm{g} \alpha}(k)\right)=2 U_{\mathrm{g} \alpha}(k)-U_{\mathrm{g} \alpha}(k-1)
\end{aligned}
$$

If the grid side voltage has the same sampling interval in four consecutive sampling moments, that is:

$$
\begin{aligned}
& U_{\mathrm{g} \alpha}(k+2)-U_{\mathrm{g} \alpha}(k+1)=U_{\mathrm{g} \alpha}(k+1)- \\
& U_{\mathrm{g} \alpha}(k)=U_{\mathrm{g} \alpha}(k)-U_{\mathrm{g} \alpha}(k-1)
\end{aligned}
$$

By Equation (15) and (16), at $k+1$ moment grid side voltage expressions is:

$$
\begin{aligned}
& \hat{U_{\mathrm{g} \alpha}}(k+1)=U_{\mathrm{g} \alpha}(k)+\left(U_{\mathrm{g} \alpha}(k+1)-\right. \\
& \left.U_{\mathrm{g} \alpha}(k)\right)+\frac{U_{\mathrm{g} \alpha}(k+2)-U_{\mathrm{g} \alpha}(k+1)}{2} \\
& =\frac{5}{2} U_{\mathrm{g} \alpha}(k)-\frac{3}{2} U_{\mathrm{g} \alpha}(k-1)
\end{aligned}
$$

In Equation(13) at $k+2$ moment the sampling current is the given value at sampling time $k$, expression is:

$$
i_{\alpha}(k+2)=i^{*}
$$

According to above analysis, through Equations (15), (17) and (18), $U_{\mathrm{g} \alpha}(k+1)$ and $i_{\alpha}(k+2)$ can be gotten. Substitute the above two results into Equation (13), we can get the output voltage $u_{\alpha}(k+1)$ in AC side at $k+1$ moment in the two-terminal flexible DC system. Finally through calculating the ratio of $u_{\alpha}(k+1)$ and high side voltage of inverter, we can get the required PWM inverter duty ratio $\mathrm{D}_{(k+1)}$.The advantages of the improved deadbeat control strategy mentioned in this section are as follows: (1) Compared with Equations (6) and (7), the current change will not be abrupt, the current deviation is reduced by $1 / 2$, and the current waveform is improved in a limited way;(2) without calculating current value at $k+2$ moment, current value at $k$ and $k+1$ moment are directly related;(3) Improve the system output current waveform to reduce current THD[18].(Specific THD analysis will be given in the next section.)

\section{Simulation verification and analysis}

In order to verify the method mentioned above, a simulation system is built to verify the correctness and effectiveness of the algorithm mentioned above. The main circuit parameters of the system are shown in Table 1. 
Table 1. Parameters of main circuit system.

\begin{tabular}{cc}
\hline The device & parameter value \\
\hline DC bus capacitance & $600 \mathrm{uF}$ \\
Inductance of inverter side & $6 \mathrm{mH}$ \\
Rectifier side inductance & $6 \mathrm{mH}$ \\
DC/AC model & $20 \mathrm{kHz}$ \\
AC/DC model & $20 \mathrm{kHz}$ \\
\hline
\end{tabular}

\subsection{Simulation platform construction}

The co-simulation platform applied in this section is composed of NI Labview $2014 \mathrm{sp} 1$ and Starsim software. The program can be directly imported into the controller after the verification of the program, which will greatly reduce the development time of the controller. Starsim is a simulation software based on NI Labview system. It can simulate and analyze simple power systems and new energy models such as microcircuits, transmission lines, transformers, inverters, photovoltaics and wind turbines. The method of use is very similar to Mulitisim, another NI software, but its advantage lies in its simpler interface and simpler operation. In its toolbar, there are not only commonly used transmission lines, capacitors, transformers, etc., but also some packaged photovoltaic modules, wind power motors, and inverter modules for users to use.
The working principles of the co-simulation platform are as follows. Labview 2014 sp1 collects data, improves the deadbeat control strategy and connected controllers at both ends, and writes programs of power supply controllers to the passive network. Starsim builds the main circuit of a back-to-back flexible DC transmission system, including two voltage source converters, two transformers, two inductors, and two AC distribution networks. In the host computer, the complete simulation platform is finally accomplish by executing node controls on the desktop and call simulation programs and main circuits of system through Starsim Design.

\subsection{Analysis of simulation results}

As shown in Figure 4 (a), the distortion of the grid side current can be obviously observed when applying the traditional VSC triangular wave control strategy which uses the Gauss white noise module of Ni controller to add the specified harmonic signal to the command current to simulate the complex power grid environment. Figure 4 (b) shows the analysis of the grid side current by using the Fast Fourier Transform (FFT). The third to seventh harmonic waves are significantly higher than the sum of the other harmonic waves, which seriously endanger the operation of distribution network.

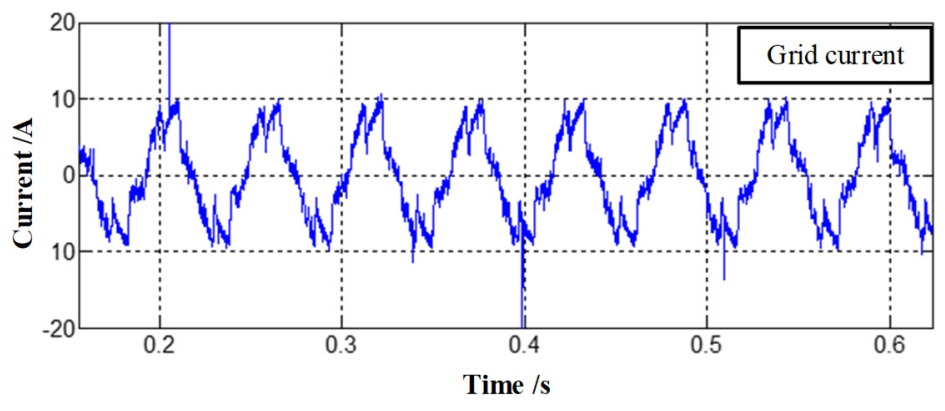

Figure 4(a). The grid side current waveform of untaken measures.

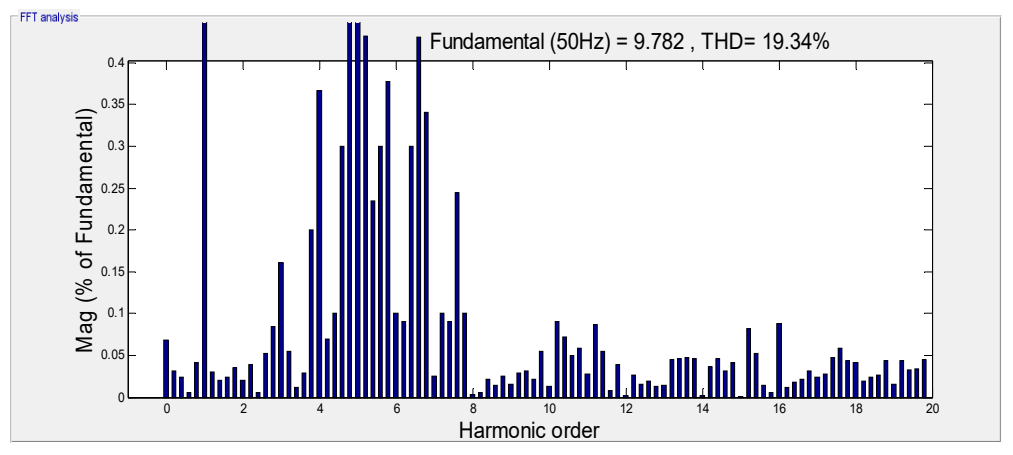

Figure 4(b). The grid side current waveform THD of untaken measures.

As shown in Fig. 5(a), traditional deadbeat control strategy is adopted. Compared with Fig. 4(a), the grid-side current waveform tends to be sinusoidal. Fig. 5(b) shows the grid-side current THD at this time. In the figure, the content of 3 to 7 harmonics is reduced, and the compensation rate of 3 to 7 harmonics is $60 \%$, but it is still high, and the operation of the distribution network is still endangered. 


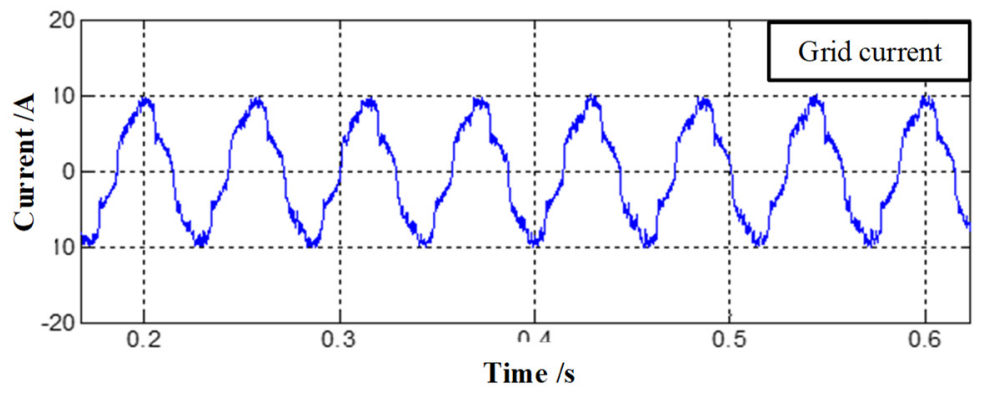

Figure 5(a). The grid side current waveform of adopt tradition deadbead.

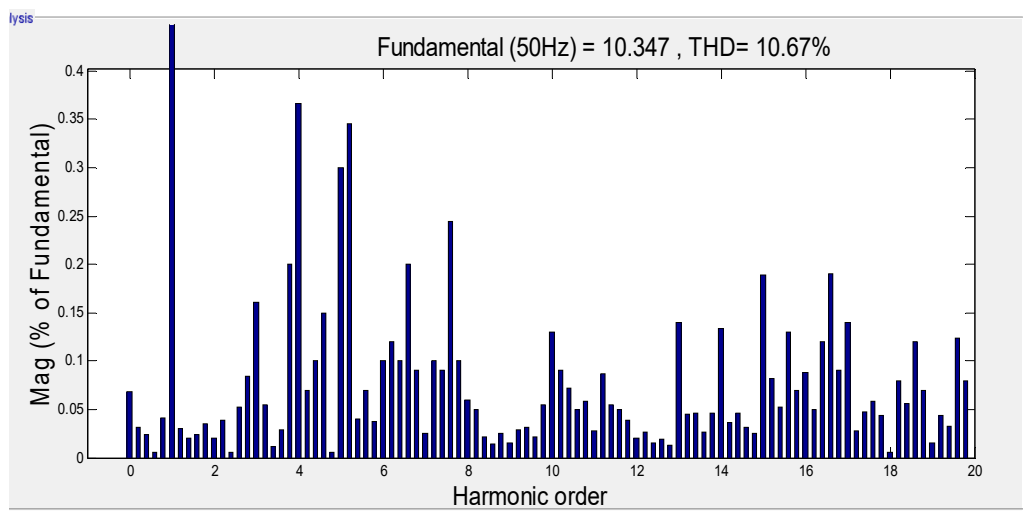

Figure 5(b). The grid side current waveform THD of adopt tradition deadbead.

As shown in Fig.6 (a) and (b), the improved deadbeat control strategy is adopted, and it can be seen from Fig.6 (b) that the current THD of the grid side is $3.69 \%$ at this time, and all the harmonics from 3 to 7 in the figure are effectively suppressed, and the compensation rate from 3 to 7 harmonics reaches $95 \%$, greatly improving the operation safety of the system.

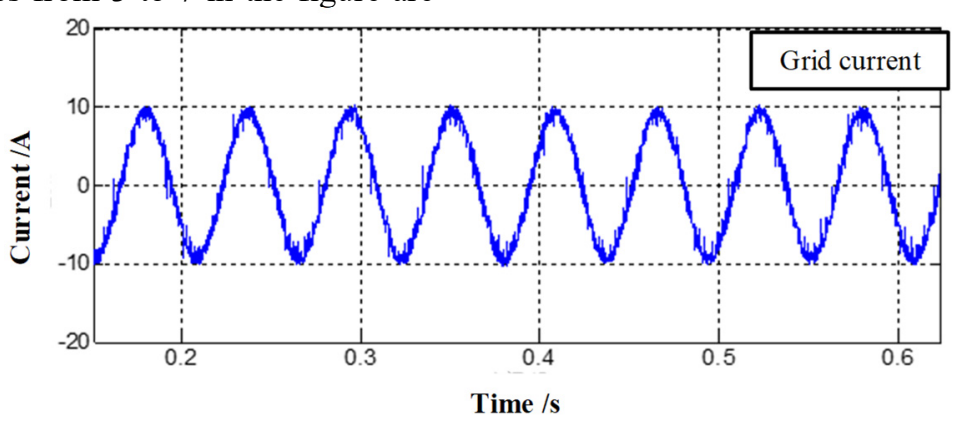

Figure 6(a). The grid side current waveform of adopt improvement deadbead.

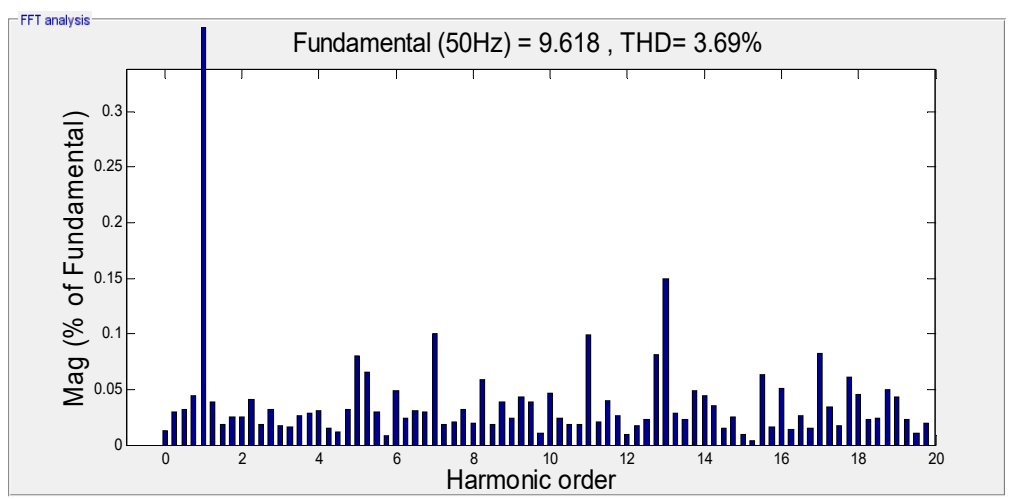

Figure 6(b). The grid side current waveform THD of adopt improvement deadbead. 


\section{Conclusion}

In this paper, a two-terminal flexible DC transmission system is taken as the research object, and a two-terminal flexible DC mathematical model is established. On this basis, the basic operation mode of the system and the control strategy of voltage source type (VSC) converter are studied. Considering the problems of control delay and poor response of current closed-loop control when traditional deadbeat control is applied to VSC converter, an improved deadbeat control strategy is proposed to solve the above defects by predicting the sampling current at $\mathrm{k}+2$ moment. Finally, the correctness and reliability of the proposed method are verified by co-simulation. In the future, the improved method proposed in this paper can be combined with other power quality management strategies, which not only widens the scope of application of the above method, but also improves the system stability.

\section{References}

1. Xu Z. (2008) HVDC and FACTS controllersapplications of static converters in power systems. China Machine Press, Beijing, China.

2. Cetin A, Ermis M. (2009) VSC based DSTATCOM with selective harmonic elimination. J. IEEE Trans. on Industry Applications, 45(3): 1000-1015.

3. ABB Co., LTD. (2007) The ABCs of HVDC transmission technology. J. IEEE Power \& Energy Magazine, 5(2): 32-44.

4. Zhang Lidong, Harnefors L, Nee H P. (2011) Interconnection of two very weak $\mathrm{AC}$ system by VSC-HVDC links using power-synchronization control. J. IEEE Trans. on Power Systems, 26(1): 344-355.

5. Flourentzou N, Agelidis V G, Demetriades G D. (2009) VSC-based HVDC power transmission system: an-overview. J. IEEE Trans. on Power Electronics, 24(3): 592-602.

6. Zhang G B, Xu Z, Wang G Z. (2002) Steady-state model and its nonlinear control of vsc-hvdc system. J. Proceedings of the CESS, 22(01): 18-23.

7. Ying M, Li G Y, Niu T Y, et al. (2005) Continuoustime state-space model of vsc-hvdc and its control strategy. Proceedings of the CESS, 18: 34-39.

8. Sun L X, Chen Y, Song H G, et al. (2016) Improved DC voltage droop control strategy for VSC-MTDC. J. Power System Technology, 40(04): 1037-1043.

9. Sun L X, Chen Y, Song H G, et al. (2016) Improved Voltage Droop Control Strategy for VSC-MTDC. Power System Technology, 40(04): 1037-1043.

10. Liang H F, Li G Y, Li G K, et al. (2005) Simulation study of vsc-hvdc system connected to passive network. Power System Technology, (08):45-50.
11. Mohamed Y. (2011) Suppression of low-and highfrequency instabilities and grid-induced disturbances in distributed generation inverters. J. IEEE Transactions on Power Electronics, 16(12): 3790-3803.

12. Yuan Q Y. (2005) Present state and application prospect of ultra hvdc transmission in china. J. Power System Technology, 29(14):1-3.

13. Lin J S. (1982) High Voltage Direct Current Transmission. Shanghai science and Technology Press, Shanghai, China.

14. Rahimi E, Gole A M, Davies J B, et al. (2010) Commutation Failure Analysis in Multi-Infeed HVDC Systems. J. IEEE Transactions on Power Delivery, 26(1):378-384.

15. Wang J, Zhang Y, Lin L. (2009) Coordinated recovery strategy of MIDC system after AC faults. J. Electric Power Automation Equipment, 29(10):79-82+83.

16. Guo L, Liu T, Li X. (2013) Measures inhibiting follow-up commutation failures in multi-infeed HVDC system. J. Electric Power Automation Equipment, 33(11):95-99.

17. Zhou J, Qin J, Wang Z J, et al. (2013) Application of deadbeat control with plug-in repetitive controller in active power filter. J. Transactions of China Electrotechnical Society, 28(2): 233-238.

18. Yang Y, Ruan Y, Ye B Y, et al. (2009) Deadbeat Predictive current control method for three-phase grid-connected inverters. J. Proceedings of the CSEE, 29(33): 40-46.

19. Chen H, Chen Y, Wan Q L, et al. (2015) Steadystate analysis of electric power system. China Electric Power Press, Beijing, China. 